ARTICLE

\title{
An integrated genomic and transcriptomic survey of mucormycosis-causing fungi
}

Marcus C. Chibucos ${ }^{1,2}$, Sameh Soliman ${ }^{3, \dagger}$, Teclegiorgis Gebremariam³ ${ }^{3}$.Hongkyu Lee ${ }^{3}$, Sean Daugherty ${ }^{2}$, Joshua Orvis $^{2}$, Amol C. Shetty2, Jonathan Crabtree², Tracy H. Hazen ${ }^{1,2}$, Kizee A. Etienne ${ }^{4}$, Priti Kumari², Timothy D. O'Connor 2,5, David A. Rasko ${ }^{1,2}$, Scott G. Filler ${ }^{3,6}$, Claire M. Fraser ${ }^{2}$, Shawn R. Lockhart ${ }^{4}$, Christopher D. Skory ${ }^{7}$, Ashraf S. Ibrahim ${ }^{3,6}$ \& Vincent M. Bruno ${ }^{1,2}$

Mucormycosis is a life-threatening infection caused by Mucorales fungi. Here we sequence 30 fungal genomes, and perform transcriptomics with three representative Rhizopus and Mucor strains and with human airway epithelial cells during fungal invasion, to reveal key host and fungal determinants contributing to pathogenesis. Analysis of the host transcriptional response to Mucorales reveals platelet-derived growth factor receptor $B$ (PDGFRB) signaling as part of a core response to divergent pathogenic fungi; inhibition of PDGFRB reduces Mucorales-induced damage to host cells. The unique presence of $\mathrm{Cot} H$ invasins in all invasive Mucorales, and the correlation between $\mathrm{CotH}$ gene copy number and clinical prevalence, are consistent with an important role for these proteins in mucormycosis pathogenesis. Our work provides insight into the evolution of this medically and economically important group of fungi, and identifies several molecular pathways that might be exploited as potential therapeutic targets.

\footnotetext{
${ }^{1}$ Department of Microbiology and Immunology, University of Maryland School of Medicine, Baltimore, Maryland 21201, USA. ${ }^{2}$ Institute for Genome Sciences, University of Maryland School of Medicine, Baltimore, Maryland 21201, USA. ${ }^{3}$ Division of Infectious Diseases, Los Angeles Biomedical Research Institute, Harbor-UCLA Medical Center, Torrance, California 90502, USA. ${ }^{4}$ Fungal Reference Laboratory, Mycotic Diseases Branch, Centers for Disease Control and Prevention, Atlanta, Georgia 30333, USA. ${ }^{5}$ Department of Medicine, University of Maryland School of Medicine, Baltimore, Maryland 21201, USA. ${ }^{6}$ David Geffen School of Medicine at UCLA, Los Angeles, California 90502, USA. ${ }^{7}$ National Center for Agriculture Utilization Research, USDA, Agricultural Research Service, Peoria, Illinois 61604, USA. † Present address: Department of Medicinal Chemistry, College of Pharmacy, University of Sharjah, P.O. Box 27272, Sharjah, UAE. Correspondence and requests for materials should be addressed to A.S.I. (ibrahim@labiomed.org) or to V.M.B. (email: vbruno@som.umaryland.edu).
} 
M ucormycoses are deadly human infections caused by fungi belonging to the subphylum Mucoromycotina, order Mucorales ${ }^{1,2}$. Rhizopus spp. are the most common organisms isolated from patients with mucormycosis and are responsible for $\sim 70 \%$ of all cases of this disease ${ }^{1-3}$. Mucor spp. and Lichtheimia spp. are also a significant cause of fungal infections in Europe with each causing $\sim 20 \%$ of the cases $^{4}$, while Apophysomyces spp. are common clinical isolates in India ${ }^{5}$. Fungal species currently belonging to both the Mucormycotina and Entomophthoromycotina subphyla were formerly considered to be members of the zygomycota taxon based on morphological similarities, sexual reproductive structures, life cycle and ecology. However, following reanalysis of their evolutionary relationship using molecular phylogenetic techniques, these two taxa have been separated ${ }^{6}$. While mucormycoses are often invasive infections, entomophthoramycoses, caused by members of the subphylum Entomophthoromycotina, order Entomophthorales, are only known to be superficial infections ${ }^{7}$.

The major risk factors for mucormycosis include uncontrolled diabetes mellitus in ketoacidosis, other forms of metabolic acidosis, treatment with corticosteroids, solid organ or bone marrow transplantation, neutropenia, trauma and burns (for example, wounded soldiers in Iraq and Afghanistan), malignant haematological disorders and deferoxamine therapy in patients receiving haemodialysis ${ }^{2,8,9}$. Cutaneous necrotizing soft tissue mucormycosis outbreaks in otherwise healthy individuals have also been known to follow natural disasters, as evidenced by the Apophysomyces infections, usually associated with trauma, following the tsunami that devastated Indonesia in 2004 (ref. 10) and the tornadoes that occurred in Joplin, Missouri, USA in June 2011 (ref. 11).

The standard therapy for mucormycosis includes reversal of the underlying predisposing factors (if possible), wide-spread surgical debridement of the infected area ${ }^{8}$ and antifungal therapy $y^{2,9,12}$. In the absence of surgical removal of the infected focus (such as excision of the eye in patients with rhinocerebral mucormycosis), antifungal therapy alone is rarely curative $e^{9,12}$. Even when surgical debridement is combined with high-dose amphotericin $\mathrm{B}$, the mortality associated with mucormycosis is $>50 \%$ (ref. 12). In patients with prolonged neutropenia, disseminated disease or central nervous system infection mortality is $90-100 \%$ (refs 13-15).

The unacceptably high mortality rate, limited options for therapy and the extreme morbidity of highly disfiguring surgical therapy make it imperative to look for alternative strategies to treat and prevent these infections. While the genomes of some Mucorales isolates have been sequenced, there remains a paucity of genome data and comparative analyses for this group of fungal pathogens ${ }^{11,16-25}$. In this work, we utilize an integrated genomics approach to understand the population genetics, evolution and phylogeny of this understudied group of fungi, and identify potential therapeutic targets in the pathogen and the host.

\section{Results}

Genome sequence and annotation of Mucorales. To identify both common and taxa-specific genetic elements that contribute to pathogenesis, we sequenced, assembled and annotated the genomes of 27 isolates from the order Mucorales, including representative isolates of 15 different species from nine different genera: Apophysomyces, Cokeromyces, Cunninghamella, Mucor, Rhizomucor, Rhizopus, Saksenaea, Syncephalastrum and Umbelopsis (Table 1; Supplementary Data 1). We performed the same on the genomes of three strains from the order Entomophthorales: Basidiobolus heterosporus, Basidiobolus. meristosporus and Conidiobolus incongruus (C. incongruus). Since, these species are not known to cause invasive infections in humans, a comparative analysis of these strains with Mucorales strains will help to understand the genetic requirements for invasive disease. The majority of the strains sequenced in this study are clinical isolates with the exception of the Apophysomyces elegans, Rhizopus stolonifer and B. heterosporus strains. The specific source of each strain is listed in (Supplementary Data 1). All strains were sequenced using the Illumina HiSeq2000 platform and each assembly was created de novo.

We performed structural and functional annotation of each newly sequenced genome and used these annotations to perform downstream comparative genomic analyses (Supplementary Data 2). A total of 41 genomes were included in our comparative analysis: the 30 presented here plus 11 previously published genomes: two Lichtheimia corymbifera ${ }^{16}$, two Lichtheimia ramosa ${ }^{16}$, one Mortierella alpina (M. alpina) ${ }^{26}$, one Rhizopus chinensis ${ }^{25}$, one Mucor velutinosus ${ }^{22}$, one Mucor circinelloides (M. circinelloides $)^{22}$, one Mucor racemosus (M. racemosus) $)^{22}$, one Mucor indicus (M. indicus) $)^{22}$ and the original Rhizopus delemar 99-880 (ref. 20). Among these 11 additional strains, 8 are clinical isolates while 3 (L. corymbifera CDC-B2541, M. racemosus CDCB9645 and Rhizopus microsporus var. chinensis) are environmental isolates. To prevent our analyses from being limited by varying approaches to genome annotation, we used our annotation pipeline to annotate (or re-annotate in the case of $R$. delemar 99-880) the 11 previously published genomes (Supplementary Data 2).

Phylogenetic analysis of Mucorales. To explore the phylogenetic relationship among the 41 isolates, we identified 76 single-copy core genes present in all 41 genomes and estimated the phylogeny of these organisms using PhyML v3.0 (ref. 27; Fig. 1). As expected, the three Entomopthorales genomes formed a distinct grouping separate from the other 38 genomes. M. alpina appears to be in a group by itself near the base of the tree, as does

\begin{tabular}{|c|c|c|}
\hline Genus & Species & No. of Isolates \\
\hline \multirow[t]{4}{*}{ Rhizopus } & R. oryzae & 8 \\
\hline & R. delemar & 4 \\
\hline & R. microsporus & 1 \\
\hline & R. stolonifer & 1 \\
\hline Mucor & M. racemosus & 1 \\
\hline \multirow[t]{2}{*}{ Cunninghamella } & C. bertholletiae & 2 \\
\hline & C. elegans & 1 \\
\hline \multirow[t]{2}{*}{ Apophysomyces } & A. elegans & 1 \\
\hline & A. trapeziformis & 1 \\
\hline \multirow[t]{2}{*}{ Syncephalastrum } & S. monosporum & 1 \\
\hline & S. racemosum & 1 \\
\hline Rhizomucor & R. variabilis & 1 \\
\hline Cokeromyces & C. recurvatus & 1 \\
\hline \multirow[t]{2}{*}{ Saksenaea } & S. oblongisporus & 1 \\
\hline & S. vasiformis & 1 \\
\hline Umbelopsis & U. isabellina & 1 \\
\hline \multirow[t]{2}{*}{ Basidiobolus } & B. heterosporus & 1 \\
\hline & B. meristosporus & 1 \\
\hline Conidiobolus & C. incongruus & 1 \\
\hline
\end{tabular}




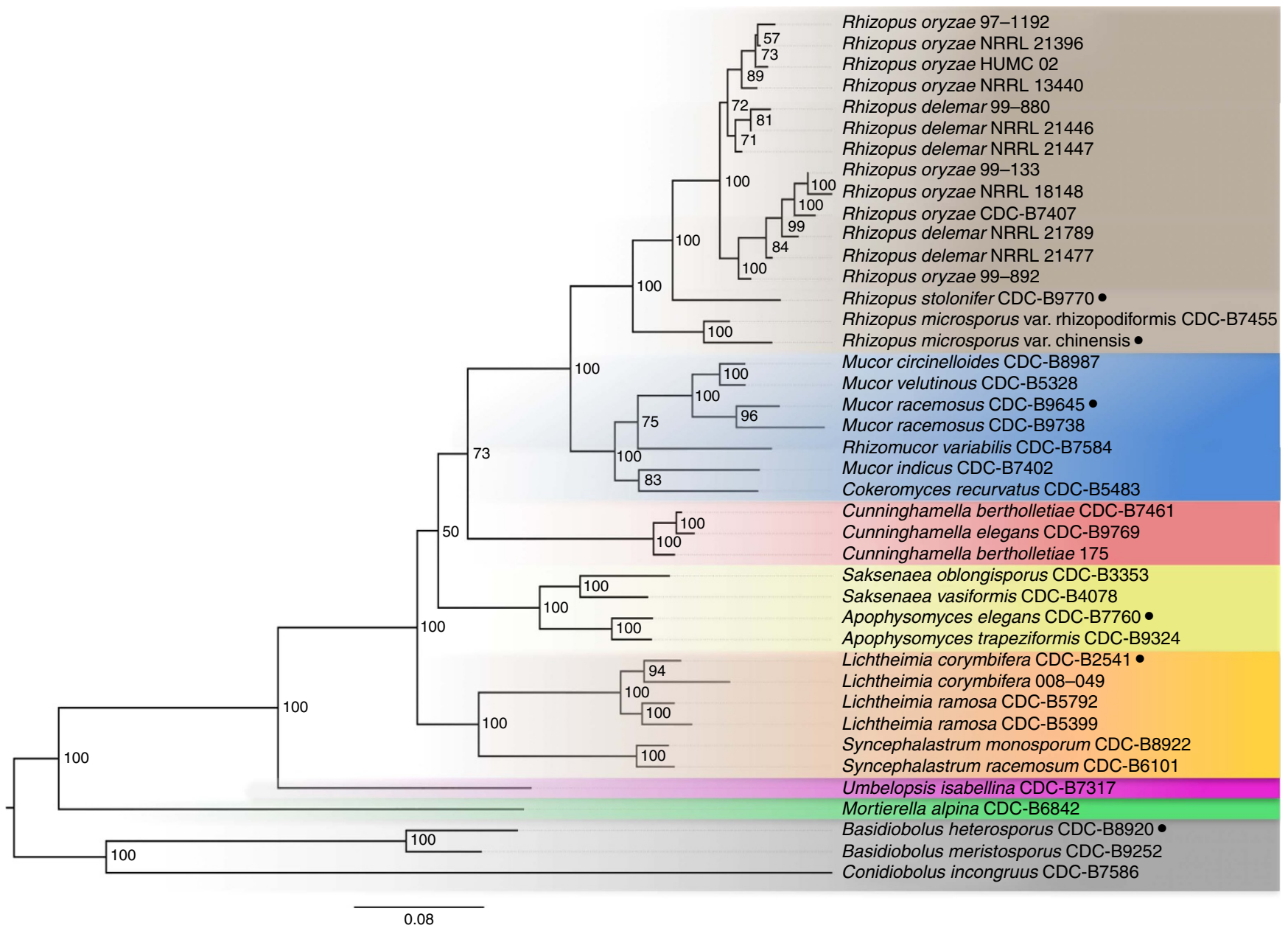

Figure 1 | Phylogenetic relationships among 38 Mucorales fungi with three Entomopthorales as outgroups. A phylogenetic tree was constructed using concatenated trimmed multiple alignments of 76 orthologous proteins shared among all 41 fungal strains. Bootstrap values (out of 100) are displayed at tree nodes. Of note, the $R$. oryzae and $R$. delemar sub-clade contains low bootstrap values throughout. All strains are clinical isolates unless otherwise indicated by a black circle to be environmental.

Umbelopsis isabellina. The remainder of the isolates, all of which are Mucorales, formed five distinct clades. One such clade was made up completely of Rhizopus isolates, while another clade included Mucor, Rhizomucor and Cokeromyces isolates. A third clade contained only Cunninghamella. Isolates of Saksenaea and Apophysomyces clustered together into a fourth clade, and the Lichtheimia and Syncephalastrum isolates formed the final clade. Overall, our results largely agree with the phylogenetic analysis performed by Hoffmann et al. ${ }^{28}$ using four molecular markers.

Unexpectedly, Mucor racemosus strain 97-1192 grouped with the Rhizopus oryzae and $R$. delemar strains, consistent with the idea that strain 97-1192 was originally misclassified as a Mucor and actually belongs to the genus Rhizopus as either oryzae or delemar. From this point forward, we will refer to strain 97-1192 as a Rhizopus strain. This strain was included in a more specific phylogenetic analysis as discussed below.

We were also surprised that $R$. microsporus strain CDC-B9738 grouped together with the Mucor and Rhizomucor isolates and, based on our analysis, is most closely related to $M$. racemosus CDC-B9645 (Fig. 1). We believe that CDC-B9738 was also misclassified and from this point forward we will refer to strain CDC-B9738 as M. racemosus.

Expansion of chitin deacetylases in Mucorales. We used an unbiased approach to identify recurring protein motifs that were present in our predicted proteomes using MEME, a program that finds novel, ungapped motifs (recurring, fixed-length patterns) in protein or nucleotide sequences ${ }^{29}$. Searching the predicted $R$. delemar 99-880 proteome with MEME revealed a number of conserved motifs, which were present throughout dozens of proteins. The sequence logos for 20 most significant motifs are depicted in Supplementary Fig. 1 and listed in Supplementary Data 3. The most significant motif discovered was annotated by our pipeline as a member of the chitin deacetylase (CDA) family of proteins (Supplementary Data 3). A hidden Markov model (HMM) built from a multiple sequence alignment of these proteins (Supplementary Fig. 2a) was searched against 92 widely divergent fungal genomes, and all except Schizosaccharomyces or Enterocytozoon had at least one peptide that was similar (Supplementary Fig. 2b). Our results are consistent with previous reports showing the expansion of this gene family in a single strain, $R$. delemar strain 99-880 (refs 20,30). However, this chitin deacetylase family also appears expanded in the other Mucoralean fungi in the present study, although it does appear restricted to the early diverging fungal lineage. The functional consequences of the CDA expansion are unclear.

Expansion of invasin-encoding genes. We have previously described the identification of three surface proteins encoded by R. delemar 99-880, CotH1, CotH2 and $\operatorname{CotH} 3$. The latter two 
function as invasins to promote infection ${ }^{31}$. We used our genomic data set to probe for the presence of CotH-like proteins in all 41 annotated genomes presented here. An HMM was built using the BLASTP hits from each genome against the CotH motif 'MGQTNDGAYRDPTDNN' (Fig. 2a). This motif is a surface-exposed region against which a therapeutic antibody has been raised $^{31}$. We then identified proteins encoded in each genome that both contained the HMM sequence and had a BLAST $e$ value of $<1 e^{-20}$. The most commonly isolated species from mucormycosis infections, Rhizopus spp., each contained 6-7 CotH gene copies. The Mucor, Rhizomucor, Cokeromyces and Lichtheimia isolates, which are also commonly isolated as the cause of mucormycosis, contained 3-7 copies depending on the isolates. The Apophysomyces, Cunninghamella, Saksenaea, Syncephalastrum, Mortierella and Umbelopsis isolates, which are rarely isolated from mucormycosis patients, contained only 1-2 copies. Lastly, the Entomopthorales isolates, which do not cause invasive disease in humans, do not contain any homologues of the CotH-like genes (Fig. 2b). Taken together, these results suggest that, when considering the genome content of Mucoralean fungi, the presence and number of CotH-like genes correlates with clinical prevalence and the ability to cause invasive disease. Future studies will evaluate the relationship between the number of CotH genes in the genomes and the ability to invade and damage host cells.

Using this genomic approach, we have identified three additional $\mathrm{Cot} H$ paralogues in the $R$. oryzae and $R$. delemar isolates. These paralogs are named CotH6 (IGS-990-880_ 03186) ${ }^{20}$, CotH7 (IGS-990-880_09445) ${ }^{20}$ and CotH8 (IGS990-880_11474 $)^{20}$ because we previously identified CotH4 and CotH5 (ref. 31). The CotH4 and CotH5 were identified by their relative low identity to the entire $\mathrm{CotH} 3$ sequence (20-24\% identity at the amino-acid level), but they lack the sequence motif 'MGQTNDGAYRDPTDNN' used in this study. The messenger RNA (mRNA) expression levels of CotH1-3 and CotH6-8 genes in $R$. delemar 99-880 and $R$. oryzae 99-892 were examined using a

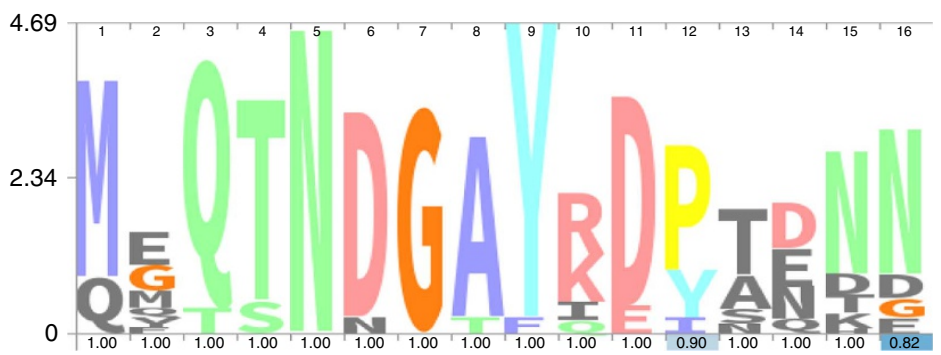

b

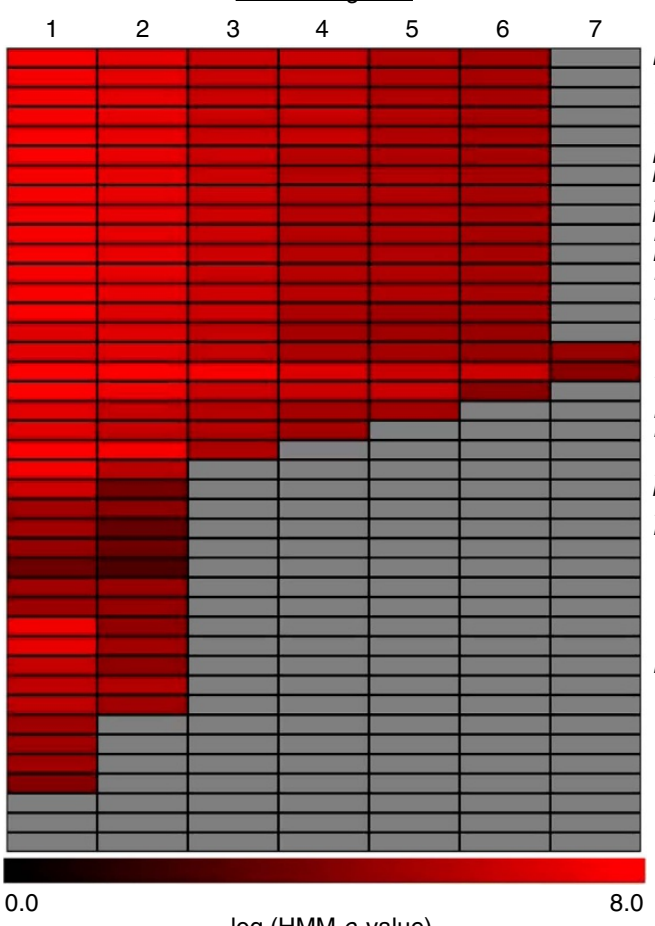

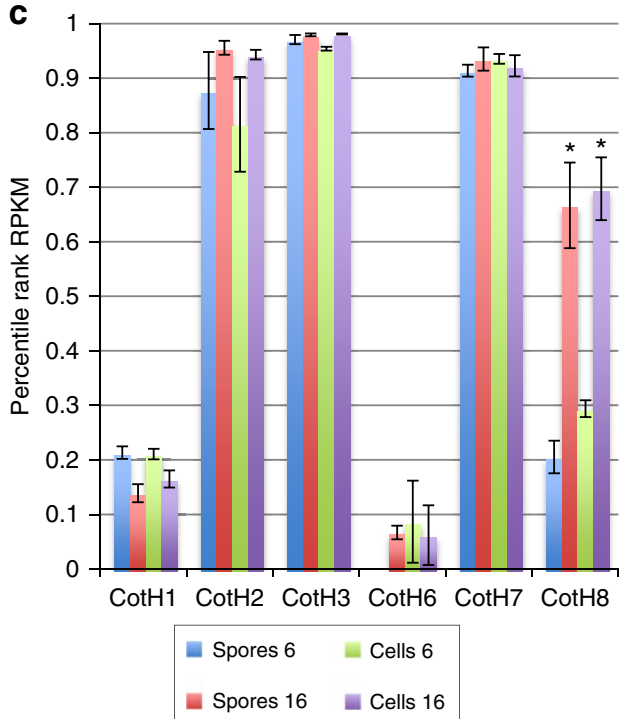

d

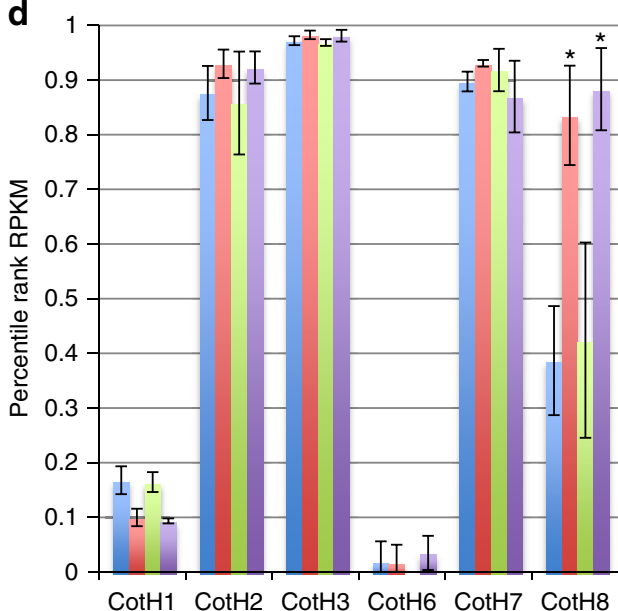

Figure 2 | Presence of CotH invasins across Mucorales. (a) HMM logo depicting conserved invasin domain. (b) Presence of CotH3 domain containing proteins in all 41 Mucorales fungi. Values represent -log of the e value from an HMM search of each positive hit (maximum e value of $1 \times 10^{-4}$ ) versus the motif from a. Grey colour indicates no gene. (c,d) Relative gene expression levels (expressed as percentile rank RPKM for all of the genes in the genome) for the six CotH genes of $R$. delemar 99-880 (c) and R. oryzae 99-892 (d) during in vitro infection conditions. Blue and red bars indicate spores grown alone in tissue culture media for $6 \mathrm{~h}$ (blue) or $16 \mathrm{~h}$ (red). Green and purple bars indicate spores grown exposed to airway epithelial cells for $6 \mathrm{~h}$ (green) or $16 \mathrm{~h}$ (purple). Results are the mean \pm s.d. ${ }^{\star} P<0.05$ versus 6 -h sample in the same condition; $n=3$. Gene of the same name in R. delemar $99-880$ and $R$. oryzae 99-892 represent 1-to-1 homologues. 
RNA sequencing (RNA-seq) data from these strains grown in the presence or absence of host airway epithelial cells. While none of the six genes are specifically induced by the presence of host cells, three of the six CotH-like genes (CotH2, CotH3 and CotH7) are among the most highly expressed genes in the genome in all four conditions, and a fourth (CotH8) seems to increase in expression with extended time in tissue culture media. Specifically, during the 6 tol6-h assay period, the R. delemar CotH8 gene is induced 3.2-fold and 2.3-fold in the absence and presence of host cells, respectively. Similar results were obtained for $\mathrm{R}$. oryzae CotH8 gene with induction of 2.1-fold and 2-fold in the same comparisons (Fig. 2c,d). The high expression of these unique $\mathrm{CotH}$ genes attests to their importance in Mucorales.

Population structure of Rhizopus strains. $R$. oryzae (syn. Rhizopus arrhizus) was reclassified into $R$. oryzae and $R$. delemar based on the ability to produce lactic acid and fumaric-malic acids, respectively ${ }^{32}$. The difference in the small organic acid production between these groups lies in the fact that $R$. oryzae strains contain a copy of LdhA, encoding lactate dehydrogenase, while $R$. delemar strains lack a copy of $\mathrm{LdhA}^{32}$. However, it has been argued that these differences are insufficient to support separate species and that varieties $R$. arrhizus var. arrhizus and var. delemar should instead be used ${ }^{33,34}$. For the sake of clarity, we use the previous nomenclature proposed by Abe et al. ${ }^{32}$ Our phylogeny presented in Fig. 1, based on a limited number of proteins provided poor resolution of the $R$. oryzae and $R$. delemar strains as evidence by the low bootstrap values. To better resolve the population structure, we performed whole-genome phylogeny for all of our $R$. delemar and $R$. oryzae genome sequences, including reclassified strain 97-1192. In total, 2,492,513 single-nucleotide polymorphisms (SNPs) were detected among all 13 isolates, of which 1,620,389 filtered SNPs present in all genomes were retained for tree building. Figure 3 a depicts the SNP-based whole-genome phylogeny. Interestingly, the isolates formed three clades instead of two clades as previous phylogenies have suggested ${ }^{32,35}$ (Fig. 3a). As expected, one clade contained only $R$. oryzae, while another clade contained only $R$. delemar isolates. The new third clade contained one strain that has been classified as $R$. oryzae, one strain classified as $R$. delemar and strain 97-1192 (formerly M. racemosus). Henceforth, we will refer to strains 99-133, NRRL 18148 and 971192 as $R$. oryzae. The possibility of two cryptic species within the $R$. oryzae group was previously recognized with phylogenic analysis of ITS, EF- $1 \alpha$ and AFLP, but not act1; however, the lack of phenotypic differences prevented them from placing species limits within this group ${ }^{32}$. The SNP-based whole-genome phylogeny more clearly defines these clades than phylogenetic analysis with single and combined gene fragments.

We next examined whether the $R$. oryzae (clade 3 ) isolates are the products of mating between $R$. oryzae and $R$. delemar strains. To address this, we performed admixture analysis on all 13 oryzae/delemar strains. The $K=2$ model, which has the lowest cross-validation error, indicates that the clade 3 individuals are
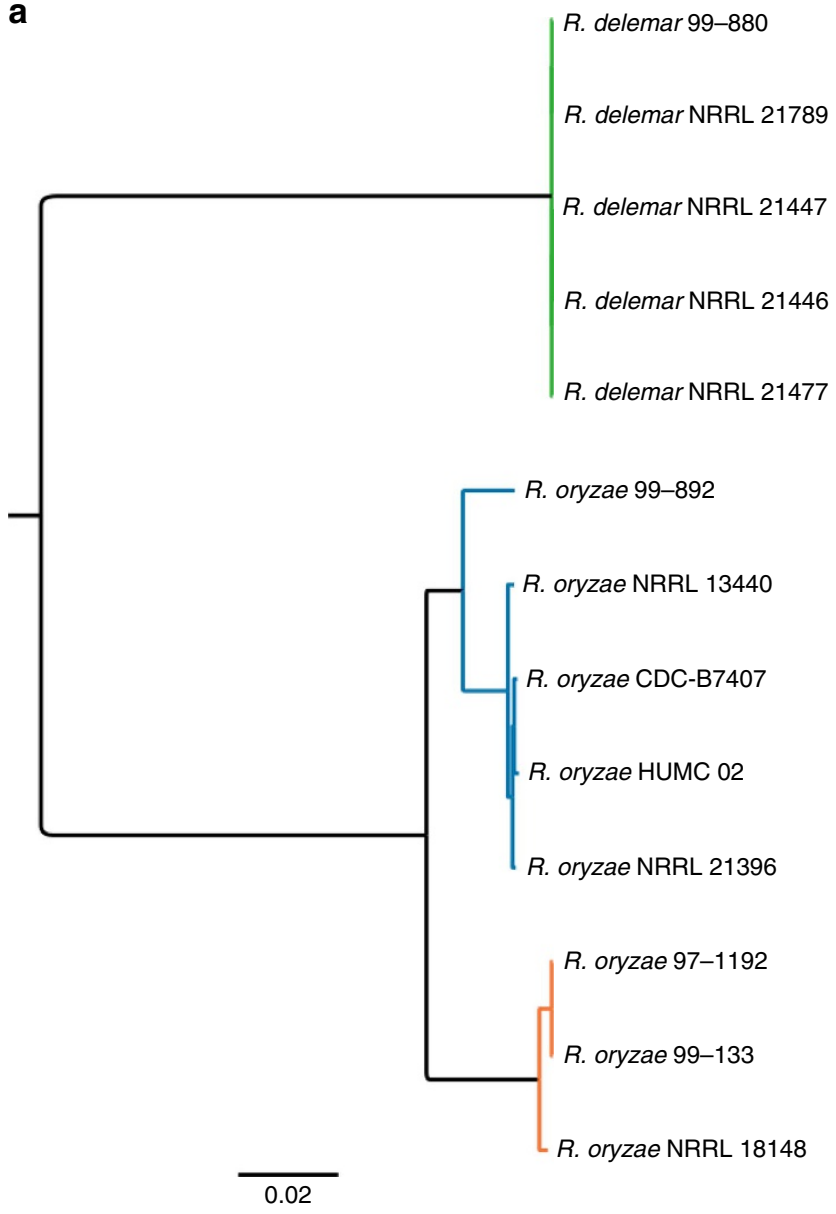

b

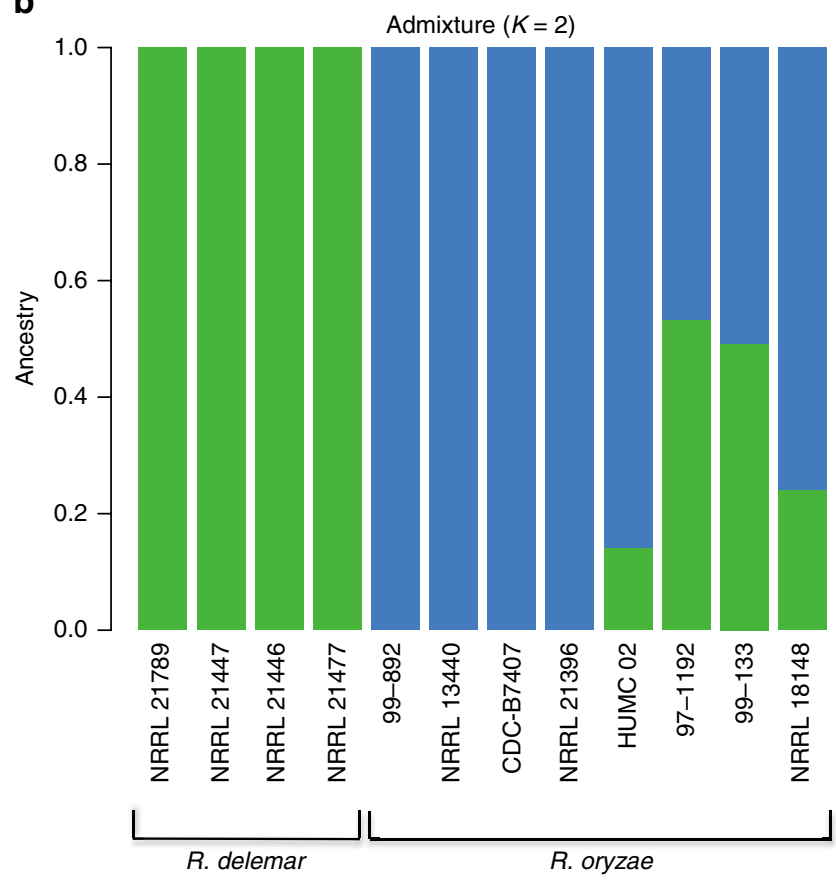

Figure 3 | Population structure of $\boldsymbol{R}$. oryzae and $\boldsymbol{R}$. delemar strains. (a) SNP-based whole-genome maximum-likelihood phylogeny of all $13 R$. delemar and $R$. oryzae strains. All nodes have a bootstrap value of $\geq 97$ out of 100. (b) Population structure inferred using the program Admixture. Values represent fraction of population ancestry denoted by colours: green ( $R$. delemar) and blue (R. oryzae). 
admixed 'mosaics' with some ancestry from each of $R$. oryzae and $R$. delemar, and that $R$. oryzae strain HUMC 02 also has some ancestry from the clade containing the $R$. delemar strains (Fig. 3b). While zygospore formation with in vitro mating has been demonstrated between $R$. oryzae and $R$. delemar ${ }^{35}$, it is unclear if zygospores germinate to produce viable progeny or if recombination has actually occurred. The small sample size warrants consideration of the $K=3$ model that only has a slightly higher cross-validation error (Supplementary Fig. 3). This model indicates a third clade, consistent with our whole-genome phylogeny, but indicates that one strain (NRRL 18148) has ancestry from both clades 1 and 3 . While both models show evidence of mixed ancestry, more information is needed to fully test these competing hypotheses.

To determine if the strains in each of the three clades vary in their virulence potential, we tested each of the strains for the ability to damage epithelial and endothelial cells during in vitro infection of monolayers. All of the strains tested, including representatives from each of the three clades, induced equivalent amounts of damage to both cell types (Supplementary Fig. 4). We also tested representatives from each of the three clades in a neutropenic mouse model of mucormycosis and observed no significant difference in the survival curves (Supplementary Fig. 5). Taken together, these results suggest that there are no clade-associated differences in the ability of these strains to cause disease.

Identification of Rhizopus-specific genes. Since R. oryzae is most commonly isolated from patients with mucormycosis, we hypothesized that its high clinical prevalence can be explained by differences in genomic content as compared with other Mucorales species. To accomplish this, we built a comparative database and searched for Jaccard orthologous clusters. Our database included the genome annotations from 13R. oryzae/ delemar strains, two $R$. microsporus strains, one $R$. stolonifer strain, one $M$. racemosus, one $L$. corymbifera strain and one Cunninghamella bertholletiae strain. We identified 2,658 'core' clusters that are present in all the 19 the genomes. To identify putative virulence genes, we took a conservative approach and searched for clusters that are present only in the 13 oryzae/delemar strains and absent from each of the other six genomes. Since, we were unable to identify any differences in pathogenic potential in vitro, we reasoned that such virulence genes should be present in all the $13 R$. oryzae/delemar strains reported here (Fig. 4). We identified 174 clusters of putative virulence genes that meet our criteria (Supplementary Data 4). We chose to further focus our list of putative virulence genes using two criteria, the presence of secretion signal and high mRNA expression, those genes whose average number of reads per kilobase of exon per million mapped reads (RPKM) ranked in the top $25 \%$ of all genes. We reasoned that genes encoding proteins with putative secretion signals might be surface exposed and directly involved in the interaction between the fungus and the host cell. Indeed, in our analyses, CotH3 has a predicted secretion signal and is known to govern pathogenesis ${ }^{31}$. Furthermore, in vivo infection studies with Candida albicans have suggested a correlation between gene expression and involvement in virulence without consideration for whether a gene's expression is induced by interaction with the host cell ${ }^{36,37}$. We acknowledge that other criteria can also be used to identify putative virulence genes; however, with the general scarcity of molecular pathogenesis data for Mucorales, we decided to use these criteria that have been demonstrated for other fungal pathogens.

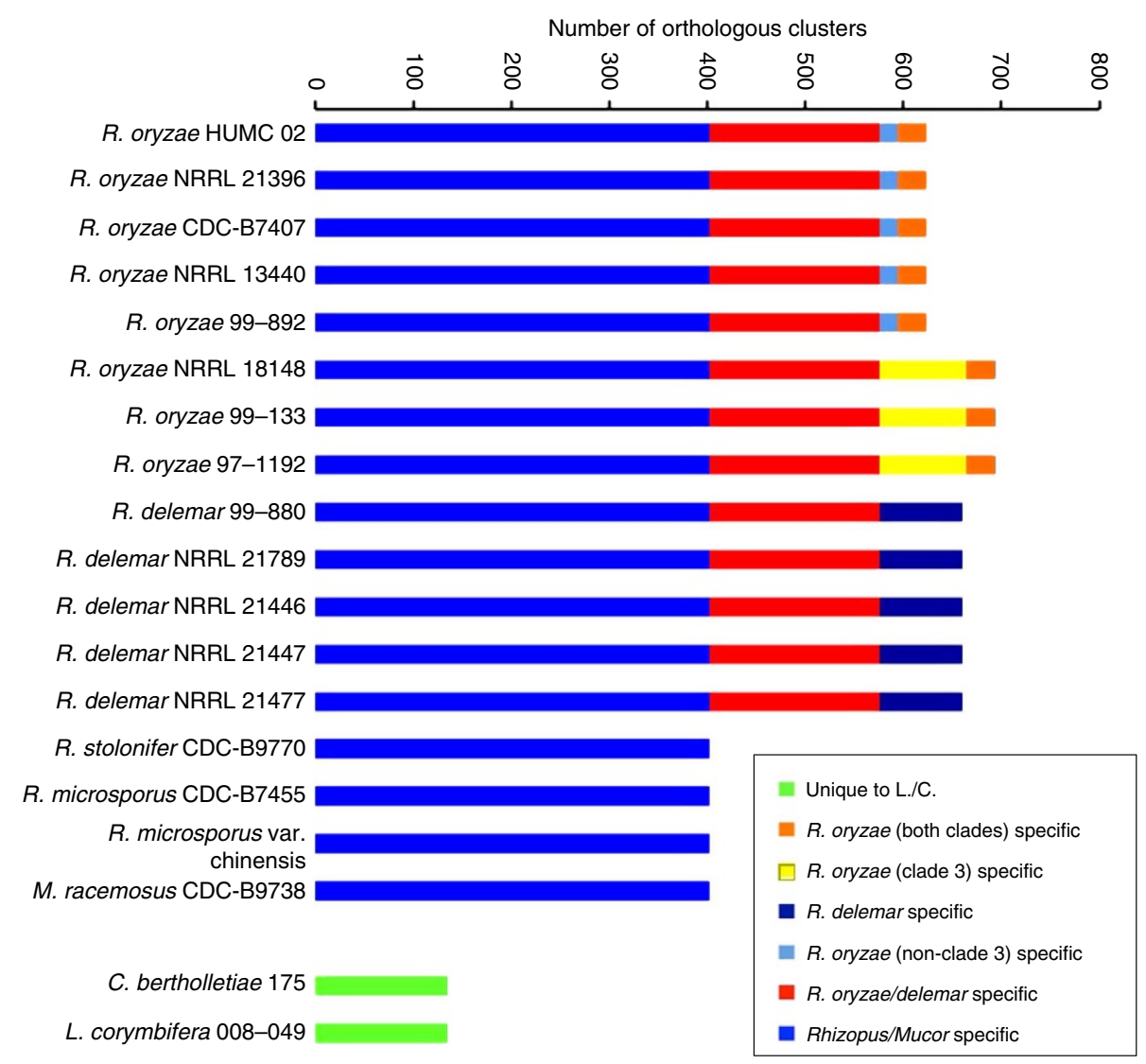

Figure 4 | Identification of orthologous clusters. A bar plot of orthologous gene clusters in a subset of 19 Mucorales fungi. Core genes found in all 17 Rhizopus and Mucor strains are shown in blue, R. delemar/oryzae-specific genes are shown in red. Please refer to key for other classifications. 
Since, we have extensive RNA-seq data sets for $R$. oryzae 99-892 and $R$. delemar 99-880 grown in the presence and absence of airway epithelial cells, we focused on these two strains. From 174 clusters, we identified six genes that meet these criteria (Supplementary Data 5). Many of the genes, including all six that have secretion signals, encode hypothetical proteins and serve as excellent candidates for virulence genes that might explain the superior clinical prevalence of $R$. oryzae and $R$. delemar. Further experiments are required to determine the role of these genes in pathogenesis.

Dual species RNA-seq of in vitro Rhizopus infection. To date, there have been no large-scale studies to examine how Mucorales fungi and host cells respond to one another during the course of infection. To this end, we performed RNA-seq on poly(A)enriched RNA isolated from human airway epithelial cells (A549) that had been infected with $R$. delemar (strain 99-880) or $R$. oryzae (strain 99-892) for 6 or $16 \mathrm{~h}$. The RNA preparations contained a mixture of mRNAs expressed by the Rhizopus spp., as well as by the host cells. To ensure that the observed fungal gene expression changes were due to the interaction with the host cell and not simply a response to the medium, we performed RNA-seq on time-matched controls in which each Rhizopus isolate was grown in each type of tissue culture medium in the absence of host cells. Similarly, we performed RNA-seq on control, uninfected airway epithelial cells.

We defined differentially expressed genes, as those with a minimum of two-fold change in gene expression $(P<0.05)$ between Rhizopus-infected host cells compared with either uninfected host cells or cells grown in the corresponding tissue culture medium in the absence of host cells. Each of the Rhizopus strains tested mounted a transcriptional response to the airway epithelial cells. $R$. delemar 99-880 displayed 459 and 583 differentially expressed genes at 6 and $16 \mathrm{~h}$ post infection, respectively (Supplementary Data 6). R. oryzae 99-892 displayed 363 and 90 genes differentially expressed at 6 and $16 \mathrm{~h}$ post infection, respectively (Supplementary Data 7). The most highly induced genes in R. delemar 99-880, at after $6 \mathrm{~h}$ of infection, are a hypothetical protein containing a motif with transporter functions. This gene (IGS-99-880_01649) displayed significant similarity to human MFSD1 (major facilitator superfamily domain containing 1). These data provide several target genes for functional follow-up experiments.

For the host analysis, in addition to $R$. orzyae 99-892 and $R$. delemar 99-880, we also examined the response of A549 cells to exposure to M. circinelloides NRRL 3631 for 6 and 16h. Analysis of host cell gene expression revealed a robust response to each of the three Mucorales strains (Supplementary Data 8), each of which induced equivalent amounts of differential host gene expression (Fig. 5a). We have previously shown that considerable insights into disease pathogenesis can be obtained by inferring signal transduction pathways based on gene expression changes ${ }^{38}$. Specifically, we used the Upstream Regulator Analytic from the Ingenuity Pathway Analysis software (Ingenuity systems; http://www.ingenuity.com) to identify signalling proteins whose downstream pathways were potentially activated or repressed during the course of infection with each of the Rhizopus strains (Supplementary Data 9). Despite some minor difference, each of the Mucorales fungi elicited similar host signalling profiles (Fig. 5b). Our analysis predicted the activation of several signalling pathways that are well established as part of the host response to fungal pathogens including: tumour necrosis factor, interleukin-1 alpha, interleukin-1 beta, nuclear factor kappa B and mitogen-activated protein kinase 1(refs 39-42). Our analysis also predicted the activation of pathways that were recently shown to be part of the host response to $C$. albicans infection including: ERBB2 (erbb2 tyrosine kinase 2), NEDD9 (neural precursor cell expressed, developmentally down-regulated 9), PDGF BB (BB homodimer), SYVN1 (synovial apoptosis inhibitor 1) and NUPR1 (nuclear protein, transcriptional regulator 1$)^{38,43}$. Given that Ascomycetes (including C. albicans) and Mucorales (including Rhizopus and Mucor spp.) are thought to have diverged from a common ancestor over $800 \mathrm{Myr}_{\text {ago }}{ }^{44-46}$, these results suggest the existence of a core host response to fungal pathogens.

We were particularly interested in the predicted activation of the PDGF BB pathway in infection because of the angioinvasive nature of mucormycosis. PDGFs are serum proteins that stimulate cellular migration and have well-established roles in angiogenesis and human diseases, such as cancer and atherosclerosis ${ }^{47-50}$. The PDGFs are encoded by four different genes-PDGFA, PDGFB, PDGFC and PDGFD_-and function as secreted homodimeric or heterodimeric proteins that bind to, and induce the tyrosine phosphorylation of, the PDGFR subunits ${ }^{47}$. The $\alpha$ and $\beta$ PDGFR subunits can combine to form homodimeric or heterodimeric receptors ${ }^{51}$. We observed a significant overlap between the set of genes that are induced in response to infection with different Mucorales strains and the set of genes that are known to be regulated by the interaction of the PDGF BB homodimer with its receptor. PDGF BB homodimers can bind to and activate signalling via the receptor $\alpha / \alpha$ homodimers, $\beta / \beta$ homodimers or $\alpha / \beta$ heterodimers ${ }^{51}$. These results are consistent with PDGFR phosphorylation and subsequent activation of cellular signalling through either the PDGFRA or PDGFRB subunits. To test whether signalling through PDGFR subunits governs the interaction between Mucorales fungi and host cells, we analysed the effects of two different small molecule PDGFR tyrosine kinase inhibitors on $R$. delemar-induced cell damage of endothelial cells. Both AG1296 (ref. 52) and PDGFR tyrosine kinase inhibitor III (CAS 205254-94-0 ${ }^{53}$ significantly reduced cell damage of endothelial cells induced by infection with $R$. delemar (Fig. 5c). These results suggest that Mucorales strains use PDGF signalling as a mechanism to damage barrier host cells and that Rhizopus likely induces expression of angiogenesis pathways to aid in its hematogenous dissemination.

Fungal cell adherence and endocytosis both contribute to the ability of Mucorales strains to cause host cell damage. PDGFR has been shown to promote endocytosis of both $C$. albicans and Chlamydia trachomatis ${ }^{54}$. While we have not specifically tested adherence or invasion in this study, further experiments are under way to elucidate the molecular mechanism underlying the reduction of damage conferred by the PDGFRB inhibitors. Although we observed significant reduction in the induction of damage upon chemical inhibition of PDGFRB, host cell damage was not completely abrogated. This might be explained by the fact that $R$. delemar is known to interact with host cell receptor GRP78 to induce its own endocytosis ${ }^{31,55}$. Another intriguing possibility is that $R$. delemar can also use ErbB2 as a receptor to induce endocytosis. On the basis of our transcriptional analysis, the ErbB2 signalling pathway is predicted to be activated by Mucorales infection. During C. albicans infection, ErbB2 signalling is predicted to be activated and inhibition of signalling reduces fungal endocytosis by the host cells ${ }^{38,43}$.

Our combination of comparative genomic and RNA-seq analyses of both fungal and host transcription during in vitro infections provides a framework for understanding the molecular pathogenesis of this increasingly important class of fungi. Specifically, we provide evolutionary insight into how pathogenesis might have evolved among this class of fungi and have identified a core host pathway that can be pharmacologically inhibited to reduce damage caused by phylogenetically distinct fungi. 
a

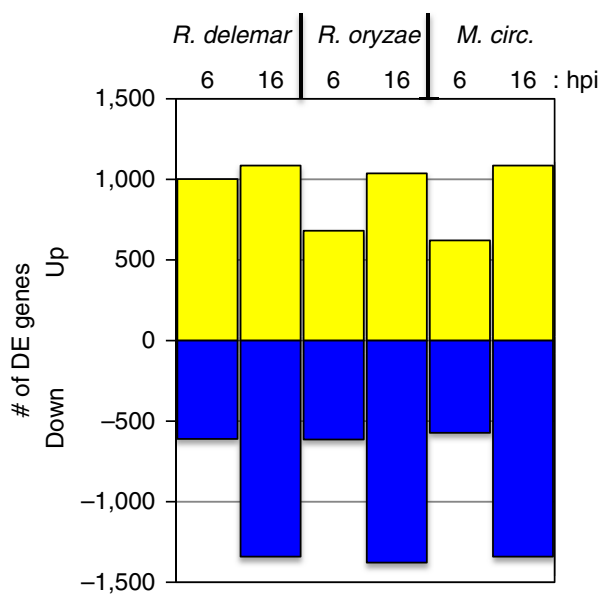

C

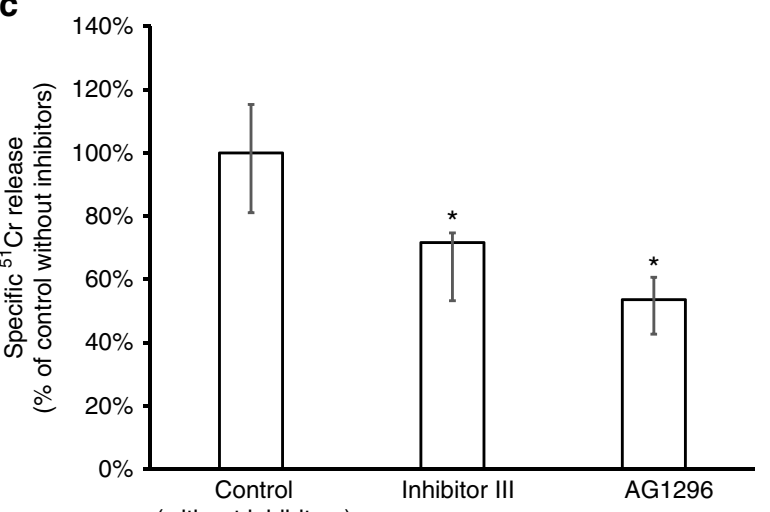

(without inhibitors) b

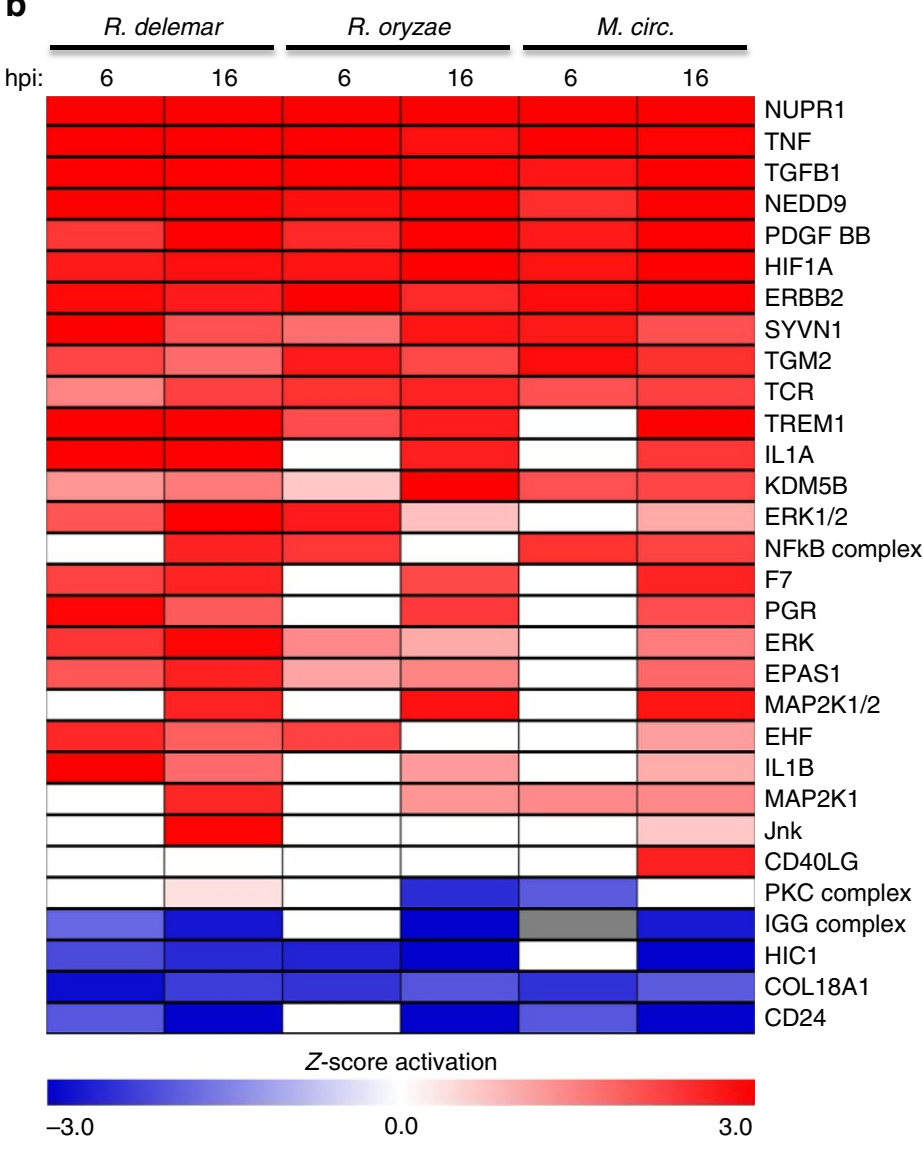

Figure 5 | Host response to Mucorales fungi. (a) The number of host genes that were induced (yellow) or repressed (blue) by infection with each of the Mucorales fungi at 6 or $16 \mathrm{~h}$ after addition of fungal spores. (b) Host upstream regulators that are predicted to be modulated by Mucorales infection of airway epithelial cells based on expression of known downstream targets. Red indicates predicted activation $(z$ score $>2)$. Blue indicates predicted repression $(z$ score $<-2$ ). White indicates no predicted regulation. (c) The effect of pharmacological inhibition of the PDFG receptor on $R$. delemar 99-880-induced cell damage of endothelial cells. Results are the median \pm interquartile range of two experiments, each performed in triplicate. ${ }^{\star} P<0.018$ versus control; $n=6$.

\section{Methods}

Strains and genome sequencing. In this study, we have sequenced, assembled and annotated the genomes of 30 fungal isolates (Table 1; Supplementary Data 1); we annotated two additional isolates sequenced previously by others $(R$. delemar 99-880 and R. microsporus var chinensis); and we included in comparative analyses nine isolates previously published by us (Supplementary Data 1). Taxa included: $A$. elegans CDC-B7760, Apophysomyces trapeziformis CDC-B9324, B. heterosporus CDC-B8920, B. meristosporus CDC-B9252, Cokeromyces recurvatus CDC-B5483, C. incongruus CDC-B7586, C. bertholletiae CDC-B7461, C. bertholletiae 175 , Cunninghamella elegans CDC-B9769, L. corymbifera 008-049 (ref. 16), L. corymbifera CDC-B2541 (ref. 16), L. ramosa CDC-B5792 (ref. 16), L. ramosa CDC-B5399 (ref. 16), M. alpina CDC-B6842 (ref. 26), M. circinelloides CDC-B8987 (ref. 22), M. indicus CDC-B7402 (ref. 22), M. racemosus CDC-B9645 (ref. 22) Mucor velutinous CDC-B5328 (ref. 22), Rhizomucor variabilis CDC-B7584, R. delemar 99-880 (ref. 20), R. delemar NRRL 21446, R. delemar NRRL 21447, $R$. delemar NRRL 21477, R. delemar NRRL 21789, R. microsporus CDC-B7455, M. racemosus CDC-B9738, $R$. microsporus var chinensis (ref. 25$)$, $R$. oryzae $99-892, R$. oryzae CDC-B7407, $R$. oryzae HUMC $02, R$. oryzae NRRL 13440, R. oryzae NRRL 21396, R. stolonifer CDC-B9770, Rhizopus species 97-1192, Rhizopus species 99-133, Rhizopus species NRRL 18148, Saksenaea oblongisporus CDC-B3353, Saksenaea vasiformis CDC-B4078, Syncephalastrum monosporum B8922, Syncephalastrum racemosum $\mathrm{B} 6101$ and U. isabellina B7317 (ref. 16).

DNA was extracted from fungi grown on Sabouraud's dextrose agar using the GeneRite kit (Carlsbarg, CA) or the OmniPrep kit (GBiosciences). The genome sequence of each isolate was generated at the Institute for Genome Sciences (IGS) Genomics Resource Center (Baltimore, MD; http://www.igs.umaryland.edu) using a combination of paired-end libraries (average insert size of $459 \mathrm{bp}$ ) and mate-pair $(3 \mathrm{~kb})$ libraries on the Illumina HiSeq2000. Draft genomes were assembled using the MaSuRCA v.1.9.2 genome assembler ${ }^{56}$.
Structural and functional genome annotation. Prediction of gene structures and assigning putative functions were performed at the IGS Informatics Resource Center (Baltimore, MD). Five isolates were grown in the presence of epithelial cell line (A549 adenocarcinomic human alveolar basal cells), human umbilical vein endothelial cells or in mammalian tissue culture media alone, and for each the following number of RNA-seq reads were generated: L. corymbifera 008-049, 439 million (previously described in Chibucos, et al. ${ }^{16}$ ); C. bertholletiae 175, 409 million; $R$. oryzae (clade 3) 97-1192 (formerly Mucor), 477 million; $R$. delemar 99-880, 412 million; and $R$. oryzae 99-892, 459 million. RNA-seq reads for a given strain were pooled and RNA-seq assemblies, both de novo and genome-guided against genomic scaffolds, were generated with Trinity ${ }^{57}$. Both types of assemblies were mapped to their respective genomes (for example, $R$. delemar 99-892 RNA-seq reads against $R$. delemar 99-892 genome) using PASA ${ }^{58}$, and de novo assemblies were mapped to other closely related genomes (for example, $R$. delemar 99-880 against the other $R$. delemar genomes) with Genomic Mapping and Alignment Program ${ }^{59}$. Genomic repeat regions were annotated and masked using RepeatMasker to detect repeats from a database of known repeats and RepeatModeler along with RepeatMasker to discover repeats de novo. Protein-coding genes were predicted $a b$ initio with CEGMA, GeneMark-ES, Augustus, SNAP, GlimmerHMM and GeneID. Augustus, SNAP and GlimmerHMM used CEGMA predictions for parameter training, and GeneID used a parameter file generated by CEGMA. Raw RNA-seq reads were used to augment Augustus training for C. bertholletiae 175, L. corymbifera 008-049 (previously described in Chibucos, et al. ${ }^{16}$ ), Rhizopus species 97-1192 (formerly Mucor), $R$. delemar 99-880 and R. oryzae 99-892. Spliced alignments of SwissProt proteins against each genome were generated with AAT using cutoffs of $80 \%$ similarity and 1,500-bp max intron length. To generate a consensus gene model set, all intrinsic and extrinsic predictions were combined with Evidence Modeller using the following evidence weights: CEGMA 4, Augustus 4, GeneMark-ES 2,

GlimmerHMM 2, SNAP 2, GeneID 2 and AAT alignments 2. Assembled RNA-seq 
transcript alignments were weighted 10 for alignment to self (for example, $R$. oryzae 99-892 transcripts aligned with PASA to $R$. oryzae 99-892 genome), but weighted 1 when aligned to other (for example, $R$. oryzae 99-892 transcripts aligned with Genomic Mapping and Alignment Program to R. oryzae 99-133). Non-coding RNAs were predicted with tRNAScan-SE and RNAmmer.

A Jbrowse ${ }^{60}$ instance displaying gene structures and expression data (RNA-seq read coverage) was set up to enable comparison of the R. delemar 99-880 gene structures generated in this study with those of the original annotation set ${ }^{20}$. To generate functional assignments, including Gene Ontology terms and Enzyme Commission numbers, predicted proteins were compared with UniProt with BLAST $\left(e\right.$ value of $1 e^{-20}$ ) and against PFAMs/TIGRFAMs with HMM searches (using cutoff values intrinsic to each model). To identify putatively secreted proteins by the presence and location of signal peptide cleavage sites, SignalP v.4.1 (ref. 61) was run on predicted proteomes.

Phylogenetic analysis. Each of 41 predicted proteomes was searched against all the others using the NCBI blastall 2.2.21 program blastp with an $e$ value of $1 e^{-40}$ and BLOSUM62 substitution matrix. BLAST results stored as XML output $(-\mathrm{m} 7)$ were filtered with the blast_parser.pl script of the InParnoid v4.1 program $^{62}$ using a bitscore cutoff of 50, and orthologues were detected between every strain pair using InParanoid (bitscore = 50; no outgroup; no bootstrapping; other parameters default). Orthologues shared among 41 strains were determined by clustering InParanoid outputs with QuickParanoid (http://pl.postech.ac.kr/QuickParanoid/), resulting in 373 protein families. Following orthologue family detection, any families where all Rhizopus proteins were not 99 or $100 \%$ identical in length were removed, to give 76 protein families in total. Orthologous proteins were aligned using Clustal Omega to produce a gapped alignment for each orthologue group. A conserved block alignment was generated for each alignment by removing poorly aligned positions and divergent regions with Gblocks v0.91b (ref. 63), resulting in 109,895 positions $(45.8 \%$ ) remaining of the intial 240,187 positions. Trimmed individual alignments were concatenated to generate a single larger alignment. Phylogenetic analysis was performed with PhyML v3.0 (ref. 64) with the following parameters: bootstrap replicates $=100$; compute approximate likelihood ratio test $=$ no; model name $=$ LG; gamma distribution parameter $=$ estimated; 'middle' of each rate class = mean; amino-acid equilibrium frequencies = model; optimise tree topology $=$ yes; tree topology search $=$ NNIs; starting tree $=$ BioNJ; add random input tree $=$ no; optimise branch lengths $=$ yes; and optimise substitution model parameters $=$ yes. The resulting tree was visualized with FigTree v1.4.2(http://tree.bio.ed.ac.uk/software/figtree/).

For the whole-genome phylogeny of the 13 Rhizopus strains, SNPs were detected relative to the genome assembly of 99-880 using the In Silico Genotyper v.0.16.10_3 (ref. 65) as previously described ${ }^{66}$. The 1,620,389 filtered SNP sites that were identified in all genomes analysed were concatenated and used for phylogenetic analysis. A maximum-likelihood phylogeny with 100 bootstrap replicates was generated using RAxML v.7.2.8 (ref. 67) and visualized using FigTree v.1.4.2.

Protein queries. The protein motif 'MGQTNDGAYRDPTDNN', an antigenic and surface-exposed peptide from $R$. delemar 99-880 CotH3 (ref. 31), was searched initially with NCBI BLASTP using default settings against the predicted proteomes of 41 strains. Matching proteins were aligned with Muscle v3.7 and outputs were manually trimmed with AliView to include only the domain. In total 144 aligned sequences with similarity to 'MGQTNDGAYRDPTDNN' were used to build a HMM with HMMER 3.0. The model was searched against all proteomes, and resulting hits above the HMM's cutoff threshold were used to construct a heat map depicting presence of proteins containing the domain across taxa. HMM logos were generated with Skylign ${ }^{68}$ with stack height representing information content above background frequency and displayed using consensus colours.

Admixture analysis. Whole-genome sequencing reads for $12 R$. oryzae/delemar samples were aligned to the $R$. delemar 99-880 genomic reference using the BurrowsWheeler aligner (v0.7.12) alignment tool. These alignment BAM files were utilized as input for GATK (v3.1.1), which included filtering of potential PCR duplicated reads, realignment around indels and fixing mates, to identify genomic variation in each sample when compared with the 99-880 genomics reference. The SNP calling was performed jointly across all 12 samples within a single execution of the 'Unified Genotyper' variant caller provided by GATK, resulting in the computation of genotypes for 2,392,625 SNP positions across the $R$. delemar 99-880 reference genome. The multi-sample VCF file from GATK was then transformed into the PLINK binary format files using the PLINK (v1.90) tool. PLINK was then used to remove any duplicates SNP positions and perform linkage disequilibrium pruning on the 12 samples to produce a reduced set of unlinked SNPs using the parameter 'indep-pairwise 5010 0.1'. This reduced set was used as input for further admixture analysis. The ADMIXTURE (v1.23) tool was used to estimate the proportions of admixture in each of the 12 samples using default settings and varying the number of ancestral populations from 1 to 5 (that is, $k=1,2,3,4$ and 5 ancestral populations). The cross-validation errors were reviewed to determine the two best values for the number of ancestral populations, namely $k=2$ and $k=3$. The estimated admixture proportions based on $k=2$ and $k=3$ were then illustrated through stacked bar plots and compared with the phylogenetic tree constructed for the same 12 samples.
RNA-seq and gene expression analysis. All RNA-seq libraries (non-strandspecific, paired end) were prepared with the TruSeq RNA Sample Prep kit (Illumina). The total RNA samples were subject to poly (A) enrichment as part of the TruSeq protocol. A sequence of $100 \mathrm{nt}$ was determined from both ends of each complementary DNA fragment using the HiSeq platform (Illumina) per the manufacturer's protocol. Sequencing reads were annotated and aligned to the human genome reference (Ensembl GRCh38), as well as the appropriate Rhizopus genome using TopHat2(ref. 69). The alignment files from TopHat2 were used to generate read counts for each gene and a statistical analysis of differential gene expression was performed using the EdgeR package from Bioconductor ${ }^{70}$. A gene was considered differentially expressed if the $P$ value for differential expression was $<0.05$ and the absolute $\log$ (base 2)-fold change was $\geq 1$.

Jaccard clustering. The predicted proteins of 19 Mucorales strains were clustered using a two-phase protein clustering algorithm ${ }^{71}$, in which the first phase defines within-genome putative paralogs ('Jaccard clusters') and the second employs a transitive reciprocal-best-hit analysis to cluster the Jaccard clusters (rather than individual proteins) across two or more genomes. The algorithm relies on an all-vs-all BLASTP search of the 19 genomes' predicted proteins, and the default parameters were used throughout. The resulting clusters and their genomic context were then examined with Sybil ${ }^{72}$, a web-based software package whose primary aim is to facilitate the analysis and visualization of comparative genome data, with a particular emphasis on protein and gene cluster data.

\section{In vitro infections and inhibitor studies. To study the host response to}

Mucorales, we incubated the alveolar epithelial A549 cells (ATCC; CCL-185) with $R$. oryzae $99-892, R$. delemar $99-880$ or M. circinelloides. A549 cells were grown in $10 \times 15$-mm tissue culture dish in F-12k medium with L-glutamine (ATCC) supplemented with $10 \%$ bovine serum albumin until confluent. The tissue culture medium was F-12 K supplemented with L-glutamine (ATCC), and $2.5 \mu \mathrm{g} \mathrm{ml}^{-1}$ of surfactant, at which time $1 \times 0^{7}$ of each Mucorales were added to each dish for a multiplicity of infection of $\sim 1.5$ cells for every one human cell. After $6 \mathrm{~h}$, or $16 \mathrm{~h}$ of infection, the cells were scraped from the plate, and the human and fungal RNA were isolated using the Qiagen RNeasy plant kit according to the manufacturer's instructions. As a negative controls, $1 \times 10^{7}$ Mucorales were added to the tissue culture plates containing medium alone without host cells and processed in parallel. Another control included RNA extracted from A549 cells incubated in parallel without any added Mucorales.

Endothelial cells were collected from umbilical vein endothelial cells by the method of Jaffe et al..$^{73}$ and propagated as we described before ${ }^{31}$. Primary human endothelial cell collection was approved by the IRB of the Los Angeles Biomedical Research Institute at Harbor-UCLA Medical Center. Because umbilical cords are collected without donor identifiers, the IRB considers them medical waste not subject to informed consent. $R$. delemar-induced endothelial cell damage was quantified by using a chromium $\left({ }^{51} \mathrm{Cr}\right)$ release assay ${ }^{74}$. In brief, endothelial cells grown in 96-well tissue culture plates containing detachable wells were incubated with $1 \mu \mathrm{Ci}$ per well of $\mathrm{Na}_{2}^{51} \mathrm{CrO}_{4}$ (ICN, Irvine, CA) in M-199 medium for $16 \mathrm{~h}$. On the day of the experiment, the unincorporated ${ }^{51} \mathrm{Cr}$ was aspirated, and the wells were washed twice with prewarmed HBSS. PDGFR inhibitors dissolved in $0.2 \%$ DMSO in $150 \mu$ RPMI 1640 medium with L-glutamine (Irvine Scientific) were added to endothelial cells for $1 \mathrm{~h}$ (final concentration of each inhibitor was $10 \mu \mathrm{M}$ ). Next, the wells were aspirated and a fresh $150 \mu$ l of RPMI 1640 containing of the inhibitor and fungal germlings $\left(1.0 \times 10^{6}\right.$ germinated for $\left.1 \mathrm{~h}\right)$ were added and the plate incubated for $4 \mathrm{~h}$ at $37{ }^{\circ} \mathrm{C}$ in a $5 \% \mathrm{CO}_{2}$ incubator. Spontaneous ${ }^{51} \mathrm{Cr}$ release was determined by incubating endothelial cells in RPMI 1640 containing $0.2 \%$ DMSO without the inhibitors or R. oryzae. At the end of incubation period, $50 \%$ of the medium was aspirated from each well and transferred to glass tubes, and the cells were manually detached and placed into another set of tubes. The amount of ${ }^{51} \mathrm{Cr}$ in the aspirate and the detached well was determined by gamma counting. The total amount of ${ }^{51} \mathrm{Cr}$ incorporated by endothelial cells in each well equalled the sum of radioactive counts per minute of the aspirated medium plus the radioactive counts of the corresponding detached wells. After the data were corrected for variations in the amount of tracer incorporated in each well, the percentage of specific endothelial cell release of ${ }^{51} \mathrm{Cr}$ was calculated by the following formula: $(($ experimental release $\times 2)-($ spontaneous release $\times 2)) /($ total incorporation $-($ spontaneous release $\times 2)$ ). Each experimental condition was tested at least in triplicate and the experiment repeated at least once.

In vivo infection. All procedures involving mice were approved by the IACUC of the Los Angeles Biomedical Research Institute at Harbor-UCLA Medical Center according to the NIH guidelines for animal housing and care. The virulence of $R$. delemar, $R$. oryzae or $R$. oryzae (clade 3 ) was compared in the neutropenic mouse model of intratracheal infection. ${ }^{75}$ Male ICR mice (6-8-week old and weighing 23-25 g from Taconic Farms, Germantown, NY) were used in this study. Neutropenia was induced by cyclophosphamide $\left(200 \mathrm{mg} \mathrm{kg}^{-1}\right.$, intraperitoneal) and cortisone acetate $\left(500 \mathrm{mg} \mathrm{kg}^{-1}\right.$, subcutaneous) on day -2 and +3 relative to infection. This treatment regimen results in $\sim 10$ days of leukopenia (day -2 to day +7 ) with total white blood cell count dropping from $\sim 130,000 \mathrm{~cm}^{3}$ to almost no detectable leukocytes as determined by Unopette System (Becton-Dickinson 
and Co.). Mice were intratracheally infected with $2.5 \times 10^{5}$ spores of either Mucorales after sedation with ketamine and xylazine ${ }^{75}$. To protect against bacterial infection due to neutropenia, mice were treated with ceftazidime (Western Medical Supply) at $5 \mathrm{mg}$ per mouse given subcutaneously from day 0 until day +7 relative to infection.

Statistical analysis. Differences in fungi-endothelial cell interactions were compared by the non-parametric Wilcoxon rank-sum test. Comparisons with $P$ values of $<0.05$ were considered significant.

Data availability. All of the raw sequencing data from this study have been submitted to the NCBI SRA database (http://www.ncbi.nlm.nih.gov). The DNA sequencing data have been deposited under accession codes SRP029722, SRP029720, SRP030764, SRP030765, SRP030703, SRP030763, SRP029751, SRP030704, SRP029724, SRP030711, SRP030715, SRP029744, SRP029718, SRP029712, SRP030712, SRP030762, SRP029709, SRP030713, SRP029747, SRP029758, SRP029597, SRP029749, SRP029754, SRP030769, SRP029746, SRP030760, SRP029750, SRP029759, SRP029879, SRP029755, SRP030719, SRP030710, SRP029741, SRP030708, SRP029743, SRP030717, SRP029717, SRP030716, SRP030709. The RNA sequencing data have been deposited under accession code SRP077731. Refer to Supplementary Data 1 for accession codes for genome sequences. All other supporting data from this study are available within the article and its Supplementary Information files, or from the corresponding authors upon request.

\section{References}

1. Ribes, J. A., Vanover-Sams, C. L. \& Baker, D. J. Zygomycetes in human disease. Clin. Microbiol. Rev. 13, 236-301 (2000).

2. Spellberg, B., Edwards, Jr J. \& Ibrahim, A. Novel perspectives on mucormycosis: pathophysiology, presentation, and management. Clin. Microbiol. Rev. 18, 556-569 (2005).

3. Roden, M. M. et al. Epidemiology and outcome of zygomycosis: a review of 929 reported cases. Clin. Infect. Dis. 41, 634-653 (2005).

4. Skiada, A. et al. Zygomycosis in Europe: analysis of 230 cases accrued by the registry of the European Confederation of Medical Mycology (ECMM) Working Group on Zygomycosis between 2005 and 2007. Clin. Microbiol. Infect. 17, 1859-1867 (2011).

5. Chakrabarti, A. \& Singh, R. Mucormycosis in India: unique features. Mycoses 57(Suppl 3): 85-90 (2014).

6. Kwon-Chung, K. J. Taxonomy of fungi causing mucormycosis and entomophthoramycosis (zygomycosis) and nomenclature of the disease: molecular mycologic perspectives. Clin. Infect. Dis. 54(Suppl 1): S8-S15 (2012).

7. Mendoza, L. et al. Human fungal pathogens of mucorales and entomophthorales. Cold Spring Harb. Perspect. Med. 5, 1-33 (2015).

8. Sugar, A. M. in Principles and Practices of Infectious Diseases 4th edn (eds Mandell G., Bennett J. \& Dolin R.) (Churchill Livingstone, 1995).

9. Ibrahim, A. S., Edwards, J. E. J. \& Filler, S. G. in Clinical Mycology (eds Dismukes W. E., Pappas P. G. \& Sobel J. D.) (Oxford University Press, 2003).

10. Andresen, D. et al. Multifocal cutaneous mucormycosis complicating polymicrobial wound infections in a tsunami survivor from Sri Lanka. Lancet 365, 876-878 (2005).

11. Etienne, K. A. et al. Whole genome sequence typing to investigate the Apophysomyces outbreak following a tornado in Joplin, Missouri, 2011. PLoS ONE 7, e49989 (2012).

12. Kwon-Chung, K. J. \& Bennett, J. E. in Medical Mycology (eds Lea \& Febiger) 81-104 (1992).

13. Husain, S. et al. Opportunistic mycelial fungal infections in organ transplant recipients: emerging importance of non-Aspergillus mycelial fungi. Clin. Infect. Dis. 37, 221-229 (2003).

14. Marr, K. A., Carter, R. A., Crippa, F., Wald, A. \& Corey, L. Epidemiology and outcome of mould infections in hematopoietic stem cell transplant recipients. Clin. Infect. Dis. 34, 909-917 (2002).

15. Kontoyiannis, D. P., Wessel, V. C., Bodey, G. P. \& Rolston, K. V. Zygomycosis in the 1990s in a tertiary-care cancer center. Clin. Infect. Dis. 30, 851-856 (2000).

16. Chibucos, M. C. et al. The genome sequence of four isolates from the family Lichtheimiaceae. Pathog. Dis. 73, 1-5 (2015).

17. Horn, F. et al. Draft genome sequences of symbiotic and nonsymbiotic Rhizopus microsporus strains CBS 344.29 and ATCC 62417. Genome Announc. 3, 1-2 (2015).

18. Lee, S. C. et al. Analysis of a food-borne fungal pathogen outbreak: virulence and genome of a Mucor circinelloides isolate from yogurt. MBio 5, e01390-01314 (2014).

19. Linde, J. et al. Sequence and genome annotation of Lichtheimia ramosa. Genome Announc. 2, 1-2 (2014).

20. Ma, L. J. et al. Genomic analysis of the basal lineage fungus Rhizopus oryzae reveals a whole-genome duplication. PLoS. Genet. 5, e1000549 (2009).
21. Schwartze, V. U. et al. Gene expansion shapes genome architecture in the human pathogen Lichtheimia corymbifera: an evolutionary genomics analysis in the ancient terrestrial mucorales (Mucoromycotina). PLoS. Genet. 10, e1004496 (2014)

22. Shelburne, S. A. et al. Implementation of a pan-genomic approach to investigate holobiont-infecting microbe interaction: a case report of a leukemic patient with invasive Mucormycosis. PLoS ONE 10, e0139851 (2015).

23. Takeda, I. et al. Genome sequence of the Mucoromycotina fungus Umbelopsis isabellina, an effective producer of lipids. Genome Announc. 2, e00071-14 (2014).

24. Tang, X. et al. Complete genome sequence of a high lipid-producing strain of Mucor circinelloides WJ11 and comparative genome analysis with a low lipid-producing strain CBS 277.49. PLoS ONE 10, e0137543 (2015).

25. Wang, D., Wu, R., Xu, Y. \& Li, M. Draft genome sequence of Rhizopus chinensis CCTCCM201021, used for brewing traditional chinese alcoholic beverages. Genome Announc. 1, e0019512 (2013).

26. Etienne, K. A. et al. Draft genome sequence of Mortierella alpina isolate CDC-B6842. Genome Announc. 2, e01180-13 (2014).

27. Guindon, S., Delsuc, F., Dufayard, J. F. \& Gascuel, O. Estimating maximum likelihood phylogenies with PhyML. Methods. Mol. Biol. 537, 113-137 (2009).

28. Hoffmann, K. et al. The family structure of the Mucorales: a synoptic revision based on comprehensive multigene-genealogies. Persoonia 30, 57-76 (2013).

29. Bailey, T. L., Johnson, J., Grant, C. E. \& Noble, W. S. The MEME suite. Nucleic Acids Res. 43, W39-W49 (2015)

30. Battaglia, E. et al. Carbohydrate-active enzymes from the zygomycete fungus Rhizopus oryzae: a highly specialized approach to carbohydrate degradation depicted at genome level. BMC Genomics 12, 38 (2011).

31. Gebremariam, T. et al. CotH3 mediates fungal invasion of host cells during mucormycosis. J. Clin. Invest. 124, 237-250 (2014).

32. Abe, A., Oda, Y., Asano, K. \& Sone, T. Rhizopus delemar is the proper name for Rhizopus oryzae fumaric-malic acid producers. Mycologia 99, 714-722 (2007).

33. Dolatabadi, S., de Hoog, G. S., Meis, J. F. \& Walther, G. Species boundaries and nomenclature of Rhizopus arrhizus (syn. R. oryzae). Mycoses 57(Suppl 3): 108-127 (2014).

34. Ellis, J. J. Species and varieties in the Rhizopus arrhizus-Rhizopus oryzae group as indicated by their DNA complementarity. Mycologia 77, 243-247 (1985).

35. Gryganskyi, A. P. et al. Structure, function, and phylogeny of the mating locus in the Rhizopus oryzae complex. PLoS ONE 5, e15273 (2010).

36. Bruno, V. M. et al. Transcriptomic analysis of vulvovaginal candidiasis identifies a role for the NLRP3 inflammasome. MBio 6, 1-15 (2015).

37. $\mathrm{Xu}, \mathrm{W}$. et al. Activation and alliance of regulatory pathways in C. albicans during mammalian infection. PLoS Biol. 13, e1002076 (2015).

38. Liu, Y. et al. New signaling pathways govern the host response to C. albicans infection in various niches. Genome Res. 25, 679-689 (2015).

39. Cannom, R. R., French, S. W., Johnston, D., Edwards, Jr J. E. \& Filler, S. G. Candida albicans stimulates local expression of leukocyte adhesion molecules and cytokines in vivo. J. Infect. Dis. 186, 389-396 (2002).

40. Moyes, D. L. et al. A biphasic innate immune MAPK response discriminates between the yeast and hyphal forms of Candida albicans in epithelial cells. Cell Host Microbe 8, 225-235 (2010).

41. Orozco, A. S., Zhou, X. \& Filler, S. G. Mechanisms of the proinflammatory response of endothelial cells to Candida albicans infection. Infect. Immun. 68, 1134-1141 (2000).

42. Villar, C. C., Kashleva, H., Mitchell, A. P. \& Dongari-Bagtzoglou, A. Invasive phenotype of Candida albicans affects the host proinflammatory response to infection. Infect. Immun. 73, 4588-4595 (2005).

43. Zhu, W. et al. EGFR and HER2 receptor kinase signaling mediate epithelial cell invasion by Candida albicans during oropharyngeal infection. Proc. Natl Acad. Sci. USA. 109, 14194-14199 (2012).

44. Galagan, J. E., Henn, M. R., Ma, L. J., Cuomo, C. A. \& Birren, B. Genomics of the fungal kingdom: insights into eukaryotic biology. Genome Res. 15, 1620-1631 (2005).

45. Stajich, J. E. et al. The fungi. Curr. Biol. 19, R840-R845 (2009)

46. Taylor, J. W. \& Berbee, M. L. Dating divergences in the fungal tree of life: review and new analyses. Mycologia 98, 838-849 (2006).

47. Demoulin, J. B. \& Essaghir, A. PDGF receptor signaling networks in normal and cancer cells. Cytokine Growth Factor Rev. 25, 273-283 (2014).

48. Kohler, N. \& Lipton, A. Platelets as a source of fibroblast growth-promoting activity. Exp. Cell Res. 87, 297-301 (1974).

49. Ross, R., Glomset, J., Kariya, B. \& Harker, L. A platelet-dependent serum factor that stimulates the proliferation of arterial smooth muscle cells in vitro. Proc. Natl Acad. Sci. USA 71, 1207-1210 (1974).

50. Westermark, B. \& Wasteson, A. A platelet factor stimulating human normal glial cells. Exp. Cell Res. 98, 170-174 (1976).

51. Boor, P., Ostendorf, T. \& Floege, J. Renal fibrosis: novel insights into mechanisms and therapeutic targets. Nat. Rev. Nephrol. 6, 643-656 (2010). 
52. Kovalenko, M. et al. Phosphorylation site-specific inhibition of platelet-derived growth factor beta-receptor autophosphorylation by the receptor blocking tyrphostin AG1296. Biochemistry 36, 6260-6269 (1997).

53. Matsuno, K. et al. Potent and selective inhibitors of platelet-derived growth factor receptor phosphorylation. 1. Synthesis, structure-activity relationship, and biological effects of a new class of quinazoline derivatives. J. Med. Chem. 45, 3057-3066 (2002).

54. Elwell, C. A., Ceesay, A., Kim, J. H., Kalman, D. \& Engel, J. N. RNA interference screen identifies Abl kinase and PDGFR signaling in Chlamydia trachomatis entry. PLoS. Pathog. 4, el000021 (2008).

55. Liu, M. et al. The endothelial cell receptor GRP78 is required for mucormycosis pathogenesis in diabetic mice. J. Clin. Invest. 120, 1914-1924 (2010).

56. Zimin, A. V. et al. The MaSuRCA genome assembler. Bioinformatics 29, 2669-2677 (2013)

57. Grabherr, M. G. et al. Full-length transcriptome assembly from RNA-Seq data without a reference genome. Nat. Biotechnol. 29, 644-652 (2011).

58. Haas, B. J. et al. Improving the Arabidopsis genome annotation using maximal transcript alignment assemblies. Nucleic Acids Res. 31, 5654-5666 (2003).

59. Wu, T. D. \& Watanabe, C. K. GMAP: a genomic mapping and alignment program for mRNA and EST sequences. Bioinformatics 21, 1859-1875 (2005).

60. Skinner, M. E., Uzilov, A. V., Stein, L. D., Mungall, C. J. \& Holmes, I. H. JBrowse: a next-generation genome browser. Genome Res. 19, 1630-1638 (2009).

61. Petersen, T. N., Brunak, S., von Heijne, G. \& Nielsen, H. SignalP 4.0: discriminating signal peptides from transmembrane regions. Nat. Methods $\mathbf{8}$, 785-786 (2011).

62. Ostlund, G. et al. InParanoid 7: new algorithms and tools for eukaryotic orthology analysis. Nucleic Acids Res. 38, D196-D203 (2010).

63. Talavera, G. \& Castresana, J. Improvement of phylogenies after removing divergent and ambiguously aligned blocks from protein sequence alignments. Syst. Biol. 56, 564-577 (2007).

64. Guindon, S. et al. New algorithms and methods to estimate maximumlikelihood phylogenies: assessing the performance of PhyML 3.0. Syst. Biol. 59, 307-321 (2010).

65. Sahl, J. W. et al. The in silico genotyper (ISG): an open-source pipeline to rapidly identify and annotate nucleotide variants for comparative genomics applications. bioRxiv (2015).

66. Hazen, T. H., Kaper, J. B., Nataro, J. P. \& Rasko, D. A. Comparative genomics provides insight into the diversity of the attaching and effacing Escherichia coli virulence plasmids. Infect. Immun. 83, 4103-4117 (2015).

67. Stamatakis, A. RAxML-VI-HPC: maximum likelihood-based phylogenetic analyses with thousands of taxa and mixed models. Bioinformatics 22, 2688-2690 (2006).

68. Wheeler, T. J., Clements, J. \& Finn, R. D. Skylign: a tool for creating informative, interactive logos representing sequence alignments and profile hidden Markov models. BMC Bioinformatics 15, 7 (2014).

69. Kim, D. et al. TopHat2: accurate alignment of transcriptomes in the presence of insertions, deletions and gene fusions. Genome Biol. 14, R36 (2013).

70. Robinson, M. D., McCarthy, D. J. \& Smyth, G. K. edgeR: a Bioconductor package for differential expression analysis of digital gene expression data. Bioinformatics 26, 139-140 (2010).

71. Crabtree, J., Angiuoli, S. V., Wortman, J. R. \& White, O. R. Sybil: methods and software for multiple genome comparison and visualization. Methods Mol. Biol. 408, 93-108 (2007).

72. Riley, D. R., Angiuoli, S. V., Crabtree, J., Dunning Hotopp, J. C. \& Tettelin, H. Using Sybil for interactive comparative genomics of microbes on the web. Bioinformatics 28, 160-166 (2012).
73. Jaffe, E. A., Nachman, R. L., Becker, C. G. \& Minick, C. R. Culture of human endothelial cells derived from umbilical veins. Identification by morphologic and immunologic criteria. J. Clin. Invest. 52, 2745-2756 (1973).

74. Ibrahim, A. S. et al. Bacterial endosymbiosis is widely present among zygomycetes but does not contribute to the pathogenesis of mucormycosis. J. Infect. Dis. 198, 1083-1090 (2008).

75. Luo, G. et al. Efficacy of liposomal amphotericin B and posaconazole in intratracheal models of murine mucormycosis. Antimicrob. Agents Chemother. 57, 3340-3347.

\section{Acknowledgements}

This project was funded in part with federal funds from the National Institute of Allergy and Infectious Diseases (NIAID), National Institutes of Health (NIH), Department of Health and Human Services under contract number HHSN272200900009C. V.M.B. D.A.R., C.M.F., S.G.F. and A.S.I. were supported by U19AI110820. S.G.F. was supported by grants R01AI054928 and R01DE017088. A.S.I. was supported by grants R01 AI063503 and R41 AI115907. The endothelial cells used in these studies were isolated from human umbilical cords, which were collected by the paediatric, perinatal and mobile unit of the UCLA Clinical and Translational Science Institute at LA BioMed/Harbor-UCLA Medical Center (UL1TR000124).

\section{Author contributions}

M.C.C. performed the bioinformatic analyses and wrote the paper; S.S., T.G. and H.L. performed the Mucorales infection experiments, and isolated genomic DNA and complementary DNA; S.D., J.O., A.C.S, P.K., J.C., T.H. and T.D.O. performed the bioinformatic analyses; K.A.E. isolated the genomic DNA; D.A.R. and C.M.F. were involved in the study design; S.R.L., C.D.S., A.S.I. and V.M.B. designed the study, analysed data and wrote the paper. All authors discussed the results and commented on the manuscript.

\section{Additional information}

Supplementary Information accompanies this paper at http://www.nature.com/ naturecommunications

Competing financial interests: A.S.I. owns shares in Vitalex Biosciences, a startup company that is developing immunotherapies and diagnostics for mucormycosis. The remaining authors declare no competing financial interests.

Reprints and permission information is available online at http://npg.nature.com/ reprintsandpermissions/

How to cite this article: Chibucos, M. C. et al. An integrated genomic and transcriptomic survey of mucormycosis-causing fungi. Nat. Commun. 7:12218 doi: $10.1038 /$ ncomms12218 (2016).

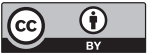

This work is licensed under a Creative Commons Attribution 4.0 International License. The images or other third party material in this article are included in the article's Creative Commons license, unless indicated otherwise in the credit line; if the material is not included under the Creative Commons license, users will need to obtain permission from the license holder to reproduce the material. To view a copy of this license, visit http://creativecommons.org/licenses/by/4.0/

(C) The Author(s) 2016 